\title{
Diamagnetic energy measurement during the first operational phase at the Wendelstein $7-\mathrm{X}$ stellarator
}

\author{
K Rahbarnia ${ }^{1}$, H Thomsen $^{1}$, U Neuner ${ }^{1}$, J Schilling ${ }^{1}$, J Geiger ${ }^{1}$, \\ G Fuchert ${ }^{1}$, T Andreeva ${ }^{1}$, M Endler ${ }^{1}$, D Hathiramani ${ }^{1}$, T \\ Bluhm $^{1}$, M Zilker ${ }^{1}$, B B Carvalho ${ }^{2}$, A Werner $^{1}$ and \\ Wendelstein 7-X Team \\ ${ }^{1}$ Max Planck Institute for Plasma Physics, Wendelsteinstr. 1, 17491 Greifswald, \\ Germany \\ ${ }^{2}$ Instituto de Plasmas e Fusão Nuclear, Instituto Superior Técnico, Lisbon, Portugal \\ E-mail: Kian.Rahbarnia@ipp.mpg.de
}

May 2018

\begin{abstract}
The magnetic diagnostic system at the Wendelstein 7-X stellarator includes three diamagnetic loops to measure magnetic flux changes in the plasma. Their signals are directly related to the plasma energy. The diagnostic design with respect to materials, component cooling and data acquisition is build to be fully steady-state capable within the harsh environment of a fusion plasma device. During the first operational phase two diamagnetic loops have been put into operation, each of them close to one of the up-down symmetric main planes of the plasma column with a bean-shaped and a triangular-shaped cross-section, respectively. Both loops measured reliably energies in accordance to theoretical expectations. The triangularshaped diamagnetic loop is equipped with four compensation coils. They are used to compensate errors during the energy measurement due to small fluctuations of externally driven currents in the main superconducting magnetic field coils and eddy currents in the adjacent vacuum vessel and thereby increase the time-resolution allowing to measure fast changes of the plasma energy. The diamagnetic flux measurements agree well with corresponding estimations of diamagnetic signals using three-dimensional Biot-Savart calculations. A consistency check for the diamagnetic energy is performed by a reconstruction of the associated Pfirsch-Schlüter current distribution and a comparison of predicted signals with measurements of an arrangement of eight plasma encircling Rogowski coil segments. Additionally, the measured diamagnetic energy is compared to kinetic energy calculations based on density and temperature measurements performed by the Thomson scattering diagnostic and the $\mathrm{X}$-ray imaging crystal spectrometer diagnostic. The resulting energy confinement times are similar to predictions of empirical scaling laws, like ISS04. For upcoming operational periods of Wendelstein 7-X the diamagnetic energy measurement will be used to generate an interlock signal, which will turn off the main plasma heating systems in case of a sudden, unwanted plasma collapse.
\end{abstract}

Keywords: stellarator, diamagnetic energy, plasma current 


\section{Introduction}

The superconducting stellarator Wendelstein 7-X (W7-X) in Greifswald, Germany, has been built following specific design criteria to optimize the magnetic configuration $[1,2]$. This optimization includes the minimization of self-generated plasma currents. In particular the reduction of Pfirsch-Schlüter currents minimizes horizontal plasma displacement (Shafranov shift [3]) and allows to achieve fusion relevant, stiff finite-beta configurations which result in good equilibrium and stability properties. Additional optimization goals were reduced neoclassical transport, low bootstrap current and good fast particle confinement at high plasma beta $[4,5]$.

The magnetic configuration of W7-X ( 5 field periods, major radius $R \approx 5.5 \mathrm{~m}$ and minor radius $a \approx 0.5 \mathrm{~m}$ ) has a pentagonal shape when viewed from above. The flux surface shape is strongly elongated (bean-shaped) in the corners of the pentagon where in most configurations the field strength is largest and changes to a more triangular shape in the half period position in the linear part (cf. figure 1a)). The flux surfaces of the magnetic configurations, used for the first operational phase (OP1.1) of W7-X from December 2015 - March 2016, were confirmed with very high accuracy (field error $<10^{-5}$ ) [6]. The experimental programs during OP1.1 were predominantly planned to commission the machine together with operational diagnostic systems [7]. Additionally a number of experiments addressed aspects of the optimization of W7-X [8-11].

In particular basic plasma parameters, like pressure profiles, plasma energy and current distributions, are essential for studying confinement and stability properties and for the mandatory reconstruction of the MHD equilibrium for a solid understanding of these properties. Magnetic pick-up coils installed at the inside and outside of the vacuum vessel of W7-X meet these requirements by providing reliable and high quality plasma energy and current measurements [12].

A number of challenging design criteria have been considered to allow for steadystate operation of the magnetic diagnostics within the demanding environment of an advanced fusion device like W7-X [13]. The implemented pick-up coils, signal cable routing and corresponding cooling systems are adapted to the complex, skewed plasma shape and space restrictions of the vacuum vessel. Integrated materials fulfill the specifications for very low outgassing rates compatible with the ultra-high vacuum (UHV) conditions and microwave stray radiation. For long pulse operation of up to 1800 s completely new data acquisition hardware and software has been developed and put into operation (details in section 2.3).

The equilibrium magnetic diagnostic system consists of continuous Rogowski coils for measuring the net toroidal plasma current as well as saddle coils and Rogowski coil segments for estimating current density distributions. Three diamagnetic loops, located at different toroidal angles with different poloidal cross-sections, are sensitive to changes of the toroidal magnetic flux, which are directly related to the plasma energy.

This work presents a description of the diamagnetic loop and Rogowski coil setup, measurement electronics and data evaluation procedures. A compensation method, 
which has been used for determining the plasma related diamagnetic flux changes and estimating the resulting plasma energy, is introduced. Diamagnetic energy and plasma current measurements are presented for typical W7-X plasma pulses during OP1.1. The theoretically expected relation between the diamagnetic energy and the dipole moment of a corresponding Pfirsch-Schlüter current distribution is shown experimentally. In comparison to the measured diamagnetic energy an independent estimate for the kinetic energy is discussed. A summary and outlook conclude the paper.

\section{Diagnostic for determining the diamagnetic energy}

\subsection{Experimental setup and basic measurement quantities}

The set of magnetic diagnostics used in OP1.1 in W7-X consisted of three diamagnetic loops, a continuous Rogowski-coil and a set of eight segmented Rogowski-coils as shown in figure 1. Two of the diamagnetic loops are installed close to the bean-shaped plane (corners of the configuration in half module (HM) 20 and 50) and have no compensation coils, the third one is close to the mid-period position (triangular plane, HM 31) and has four compensation coils (see figure 1b) and c)). The normal direction of the planes of the diamagnetic loops have been oriented with respect to the magnetic axis as to minimize the effect of net-toroidal currents in the measurement signals. The continuous Rogowskicoil and the set of eight segmented Rogowski coils are located close to triangular planes in HM 11 and HM 21, respectively. A detailed technical description of the diagnostic setup can be found in [13].

The loops measure changes of the toroidal magnetic flux originating from the diamagnetic current flowing perpendicular to the magnetic field, which is necessary to fulfill the radial force balance of the MHD-equilibrium:

$$
\mathbf{j}_{\perp}=\frac{\mathrm{B} \times \nabla_{\perp} \mathrm{p}}{\mathrm{B}^{2}} .
$$

This expels toroidal flux from the plasma region and changes the flux gathered by the diamagnetic loop.

For estimating the magnetic flux $\Phi$, measured by the diamagnetic loops, the following equation is used [14]:

$$
\Phi=\frac{\mu_{0}^{2} I_{p l}^{2}}{8 \pi B_{0}}+\frac{\pi \mu_{0} \iota}{R} \int_{0}^{a} j_{\varphi}(r) r^{3} d r-\frac{\mu_{0} W_{d i a}}{3 \pi R B_{0}} .
$$

Here $I_{p l}$ represents the toroidal net plasma current, which is measured with a continuous Rogowski coil, $B_{0}$ is the on-axis magnetic field at the corresponding toroidal position (location of the diamagnetic loop), $a$ and $R$ are the minor and major radius, respectively, $\iota$ is the rotational transform of the magnetic configuration (assumed to be constant), $j_{\varphi}(r)$ is the toroidal component of the net plasma current density at radius $r, W_{d i a}$ the diamagnetic plasma energy and $\mu_{0}$ the vacuum permeability. For the plasma pulses investigated during OP1.1, the toroidal net plasma current is very low, $I_{p l}<3 \mathrm{kA}$ and hence the first two terms in equation 2, i.e. the so-called paramagnetic and the 
stellarator specific contributions, can be neglected. The experimental verification of this assumption is shown in section 3.1. Thus, for the initial evaluations only the remaining third term in equation 2 , the diamagnetic contribution, is used for estimating the plasma energy

$$
W_{d i a}=-3 \pi R B_{0} \Phi / \mu_{0} \text {. }
$$

For typical values of W7-X $\left(R=5.5 \mathrm{~m}\right.$ and $\left.B_{0}=2.33 \mathrm{~T}\right)$ the proportionality constant is $3 \pi R B_{0} / \mu_{0}=0.96 \cdot 10^{8} \mathrm{~J} / \mathrm{Vs}$ for a single turn of a coil. A more precise calculation for the magnetic configuration used in OP1.1 with the 3D-equilbrium code VMEC $[15,16]$ and the post-processing code DIAGNO [17] to evaluate magnetic diagnostics shows for the uncompensated signal a value of $1.06 \cdot 10^{8} \mathrm{~J} / \mathrm{Vs}$. The inclusion of the compensation coils in the signal evaluation modifies this value, but will be discussed further below in section 2.2 .

Since $\mathbf{j}_{\perp}$ is generally not divergence-free, a current parallel to the magnetic field $\left(\mathbf{j}_{\|}\right)$is required to close the current path, such that $\nabla \cdot \mathbf{j}=\nabla \cdot\left(\mathbf{j}_{\perp}+\mathbf{j}_{\|}\right)=0$. This parallel current component, the so-called Pfirsch-Schlüter current, contributes to the toroidal current density, but does not add to the toroidal net current. Due to its dipole character (the toroidal direction of the currents is different for inboard and outboard side), it generates a vertical magnetic field, which displaces the plasma towards the outboard side of the torus, the so-called Shafranov shift.

The dipole character of this pressure gradient induced current distribution can be assessed with the set of eight individual Rogowski coil segments, which are located in HM 21 as shown in figure 1(a). Whereas the toroidal position varies in the order of a few centimeters for all eight segments, their poloidal arrangement is adapted to the triangular cross section and encircles the plasma $[12,13]$. The net plasma current is measured by the continuous Rogowski coil (HM11, figure 1(a)).

\subsection{Compensation of diamagnetic signal}

The diamagnetic measurement generally suffers from various sources of unwanted flux changes, like variations in the currents of the magnetic field coils or eddy currents in the vacuum vessel, which need to be corrected in order to have a clean signal. This is usually performed by equipping the diamagnetic loop with an additional loop or set of coils not enclosing the plasma which will pick up the perturbations and which can be used to compensate and clean the diamagnetic measurement. Due to space restrictions in the vacuum vessel of W7-X only the triangular-shaped loop is surrounded by an arrangement of four individual coils (figure 1(c)). The experimental compensation method, which has been applied in the context of this work, uses the compensation coil signals as follows

$$
\Phi=\phi_{d i a}-\mathbf{C} \sum_{\mathrm{i}=1}^{4} \phi_{\text {comp }}^{\mathrm{i}},
$$

where $\Phi$ is the compensated diamagnetic flux and $\phi_{\text {dia }}$ and $\phi_{\text {comp }}^{i}$ are the signals of the diamagnetic loop and the four compensation coils, respectively, which are acquired from 
the corresponding raw signals by

$$
\phi_{\text {dia }}=c_{\text {dia }} \int_{t_{1}}^{t_{2}} U_{d i a}^{\text {raw }}(t) d t, \phi_{\text {comp }}^{i}=c_{\text {comp }}^{i} \int_{t_{1}}^{t_{2}} U_{\text {comp }}^{i, r a w}(t) d t .
$$

Here $c_{d i a}, c_{\text {comp }}^{i}$ are the corresponding electronic conversion factors from measured voltages to fluxes.

To determine the factor $\mathbf{C}$ a calibration of the diagmagnetic flux compensation has been performed during simultaneously driven current ramps within the main nonplanar and planar field coils without plasma operation (figure 2). The actual slopes of the current ramps were $29.9 \mathrm{~A} / \mathrm{s}$ and $11.7 \mathrm{~A} / \mathrm{s}$ for the non-planar and planar coils, respectively. The calibration measurement was done for a $2 \mathrm{~s}$ time period at about half maximum current values. Figure 3(a) shows the resulting integrated signals measured on December $9^{\text {th }}, 2015$ by the triangular-shaped diamagnetic loop and the compensation coils. The depicted curves clearly reflect the imposed flux change.

The absolute values agree within $8 \%$ with predicted values, which have been obtained by Biot-Savart calculations using the geometry of the diamagnetic loop and the compensation coils, and the associated vector potential of the magnetic field generated by the superconducting main field coils. The deviation is related to a known pronounced sensitivity to temperature drifts in the measurement electronics. For upcoming operational phases of W7-X this effect has been minimized by improved electronic parts within the data acquisition systems, resulting in deviations of about $1 \%$ compared to predicted values.

To obtain the calibration factor $\mathbf{C}$ in equation 4 the following condition has to be fulfilled:

$$
\phi_{\text {dia }}-\mathbf{C} \sum_{\mathrm{i}=1}^{4} \phi_{\text {comp }}^{\mathrm{i}}=0 .
$$

In figure $3(\mathrm{~b})$ the relative quantity $\mathbf{C}(\mathrm{t})=\phi_{\mathrm{dia}} / \sum_{\mathrm{i}=1}^{4} \phi_{\text {comp }}^{\mathrm{i}}$ is shown for two different calibration runs on December $9^{\text {th }}, 2015$ (derived from the measured fluxes depicted in figure 3(a)) and February $2^{\text {nd }}, 2016$. Comparing the measured to the predicted factor shows a very good agreement within $<1 \%$. In the following the resulting mean value $\mathbf{C} \approx 7.65 \pm 0.01$ is used in equation 4 .

The inclusion of the compensation coils in the signal evaluation changes in principle the simple formula used in section 2.1 (equation 3). However, the proportionality between plasma energy and measured magnetic flux is still valid, although the proportionality constant may be changed. Nevertheless, VMEC/DIAGNO calculations evaluating the combination of the diamagnetic loop and compensation coils reproduce in the OP1.1-configuration the proportionality constant $0.96 \cdot 10^{8} \mathrm{~J} / \mathrm{Vs}$ in equation 3 .

\subsection{Data acquisition systems}

The long-pulse operation foreseen for W7-X (up to $1800 \mathrm{~s}$ ) and the low signal amplitudes due to strongly reduced plasma currents lead to very demanding requirements for the 
data acquisition systems (DAQ). Therefore the magnetic diagnostics are equipped with specifically designed data stations based on ATCA (Advanced Telecommunications Computing Architecture) ADC (analog to digital converter module) boards both designed and produced by the Instituto de Plasmas e Fusão Nuclear, Instituto Superior Técnico, Lisbon. Technical details and descriptions of performed tests can be found in [18]. In total $10 \mathrm{DAQ}$ stations exist, each consisting of a 32-channel ATCA carrier board, data collector PC and timing device. Each individual measurement channel is equipped with a phase modulated ADC adapted to the expected input voltage dynamic range of the connected sensor. The input signal is by default chopped with a frequency of $2 \mathrm{kHz}$ at an early point of the signal path, which mitigates the vulnerability of the measurement to offset level drifts and error voltages for long plasma pulses [19].

Figure 4 shows an example raw signal of the diamagnetic loop located in the triangular cross section. In the upper right zoomed insert the effect of the hardwareimplemented signal chopping is visible. For further data processing the raw data is de-chopped by software (lower right insert) based on the recorded chopper clock signal. For obtaining the magnetic flux change in units of Vs, the processed data are numerically integrated and corrected for offsets and gain factors of the corresponding electronics (as indicated for the diamagnetic loop and compensation coils in equation 5). The very low noise level of the data acquisition allows both the measurement of the diamagnetic energy and of the plasma current to be sensitive to very small amplitude variations of the order of $1 \mathrm{~kJ}$ and $10 \mathrm{~A}$, respectively.

\section{Discussion of experimental results}

\subsection{Measurement of the compensated diamagnetic energy}

During the second half of the experimental phase OP1.1 the compensated diamagnetic energy has been measured and evaluated routinely and was stored in the W7-X archive data base. To demonstrate the excellent signal quality and necessity for the energy signal compensation by means of the four additional compensations coils, described in section 2.2, two plasma pulses have been chosen. Both discharges are heated with a similar power of almost $2 \mathrm{MW}$ (figure 5(a)) using the W7-X electron cyclotron resonance heating $(\mathrm{ECRH})$ system [20]. They show very similar electron temperatures in the plasma center of about $8 \mathrm{keV}$ (measured with a central channel of the electron cyclotron

emission diagnostic [21]) and also similar line-integrated densities of almost $1.5 \cdot 10^{19} \mathrm{~m}^{-2}$ (measured with the single channel dispersion interferometer [22]). In these two cases the ECRH is launched with two different launching angles to drive a toroidal plasma current via electron cyclotron current drive (ECCD) [20], which points either in the direction of the toroidal bootstrap current (co-ECCD) or in the opposite direction (counter-ECCD). Thus in case of co-ECCD the low intrinsic toroidal net current is enhanced and during counter-ECCD it is reduced. The measurement of the toroidal plasma current with the continuous Rogowski coil clearly reflects the difference in the current drive scenarios 
with a reduction of the total net current to almost zero (figure 5(a)) for counter-ECCD and a positive current of up to $1.8 \mathrm{kA}$ for co-ECCD. A systematic error for the current measurements in OP1.1 is of the order of $10 \%$ for the same reason as mentioned in section 2.2 (pronounced sensitivity to temperature drifts in the measurement electronics).

Figure 5(b) shows four time series of the measured diamagnetic energy, which has been derived using equation 3 and equation 4 . The observed values of the uncompensated signals show reasonable mutual agreement although they are measured at two different toroidal locations with loops of different shapes (case $\mathrm{C}$ triangular plane and D close to bean-shaped plane; see also figure 1). It is noted that the residual values after turning off of the heating power completely vanish after about $6 \mathrm{~s}$. For comparison the energies derived from the compensated signals for co- and counter-ECCD (case A and B), measured by the diamagnetic loop in the triangular plane, are shown. Due to the compensation procedure non-negligible, non-plasma related residual parts of the signals, observed in the uncompensated energy signals after turning off of the heating power are removed and as expected a faster signal increase at the beginning of the plasma pulse compared to the uncompensated signal is visible. Hence, the energy compensation described in sec 2.2 successfully corrects for external flux contributions.

The temporal evolution of the diamagnetic energies for co- and counter-ECCD (case A and B) deviate less than 5\% from each other, which is expected as the plasma parameters are not significantly different. This deviation is well within the observed expectations for comparable discharges during OP1.1 without ECCD. The observed small plasma current does not affect the diamagnetic energy. As mentioned before, throughout the experimental phase OP1.1 the measured plasma current was very low $\left(I_{p l}<3 \mathrm{kA}\right)$, which justifies the use of equation 3 and equation 4 for estimating the diamagnetic energy from the compensated signals.

\subsection{Estimation of the Pfirsch-Schlüter dipole moment}

As explained earlier, the current density $\left(\mathbf{j}_{\perp}\right)$ required for balancing the kinetic and electromagnetic forces in the plasma is connected with a parallel current density to ensure zero divergence. These secondary currents can be assessed with the set of eight Rogowski coil segments measuring the poloidal field components. In the following the signals of these eight Rogowski coil segments are analysed for a plasma pulse without external current drive. The eight coils encircle the plasma as sketched in figure 6(b) and the numbers $\# 1 \ldots \# 8$ refer to the individual segments. The measured magnetic flux distribution clearly separates two regions of opposite sign for inboard (figure 6(a), bottom plot, line \#3,\#4,\#5,\#6) and outboard segment positions (figure 6(a), bottom

plot, line \#1,\#2,\#7,\#8). The signals reflect the ECR-heating power step at about $0.6 \mathrm{~s}$ (figure 6(a), top plot). It is noted (although not explicitly shown) that the magnetic flux measurement of the continuous Rogowski coil is comparable to the sum of signals of all eight individual Rogowski coil segments, considering that the radial gaps and toroidal displacements of the segments are rather small. 
The Rogowski coil segment measurements per se do not imply information on radial current density profiles. In order to visualize a possible current distribution, a signal reconstruction on a rectangular $25 \times 17$ grid in the poloidal measurement plane has been performed. The contribution of a given current filament through each grid point to the signal value in the coil segments was pre-calculated with Biot-Savart and stored in a contribution matrix. For simplicity straight current filaments perpendicular to the plane of the coil set were assumed, i.e. no toroidal effects have been included. For the measured data the distribution of the toroidal currents at the grid points was estimated by inverting the contribution matrix using singular value decomposition techniques and applying the measured signal values [23]. The expected dipole structure can be identified clearly (e.g. shown at $150 \mathrm{~ms}$ in figure 6(b)). Such a dipole pattern for the typical Pfirsch-Schlüter current distribution has also been observed elsewhere, e.g. in the Helically Symmetric Experiment (HSX), Madison, Wisconsin (USA) [24].

The compensated diamagnetic energy is now compared to the associated dipole moment $\mathbf{m}$ inferred from the Rogowski segment observations (figure 6(c), top plot). For an arbitrary current density distribution $\mathbf{j}(\mathbf{r})$ given in a volume $V$ the associated dipole moment $\mathbf{m}$ is expressed as

$$
\mathbf{m}=\frac{1}{2} \int_{V}(\mathbf{j}(\mathbf{r}) \times \mathbf{r}) \mathrm{d}^{3} \mathbf{r} .
$$

For the current density distribution estimated from the Rogowski coil segment measurements (figure 6(b)), an estimate of the dipole moment is derived by carrying out the integration in equation 7 over the volume with a cross section determined by the shape of the Rogowski coil segments and a width given by their toroidal extent, which is on the order of a few $\mathrm{cm}$. Therefore the integral can be simplified to a sum, which is proportional to the following expression:

$$
\mathbf{m}_{R S}=\frac{1}{2} \sum_{i, j}\left|\mathbf{j}_{i j}\right|\left(\mathbf{e}_{i j} \times \mathbf{r}_{i j}\right),
$$

with the current density $\left|\mathbf{j}_{i j}\right|$ on a given grid and corresponding position indices $i$ and $j$. The current density unit vector $\mathbf{e}_{i j}$ is taken as the vector perpendicular to the cross section of the Rogowski coil segments at the location of the corresponding grid cell. The position vector $\mathbf{r}_{i j}$ is taken with respect to the magnetic axis at the toroidal location of the Rogowski coil segments.

During the plasma pulse an almost constant proportionality factor $F$ between magnitude of $\mathbf{m}_{R S}$ and diamagnetic energy is found (figure 6(c), bottom plot). $F$ varies within $\pm 2 \%$ for a total number of 12 selected experiment programs from February 2016 - March 2016 during OP1.1. The indicated relation between the diamagnetic energy and the dipole moment of reconstructed current density distribution is consistent with the expectation that the current distribution is caused by the Pfirsch-Schlüter currents.

In this context advanced data analysis techniques, which are currently under development and base on MHD-equilibrium reconstructions within the MINERVA framework [25], will deliver 3D-current profiles. They are used to confirm aspects of 
the W7-X optimization, in particular the reduction of the Pfirsch-Schlüter currents [26]. Magnetic configurations, which have been specifically designed for long plasma pulse operation with expected low plasma currents, are suitable and envisaged for upcoming operational phases.

\subsection{Comparison of independently measured diamagnetic and kinetic energy}

The measured compensated diamagnetic energy has been compared to the independent estimate of the kinetic energy based on plasma density and electron temperature profile measurements with the Thomson scattering diagnostic [27,28] and ion temperature measurements with X-ray imaging crystal spectrometer diagnostic (XICS) [29]. Investigations presented in [30] describe the results of the comparison in more detail. It has been found that kinetic and diamagnetic energy agree relative to each other within about $10 \%$. The achieved energy confinement times are up to about $150 \mathrm{~ms}$ and scale predominantly with the empirical law ISS04 [30,31].

Figure $7(\mathrm{a})$ shows a quantitative comparison of kinetic and diamagnetic energy for a selected plasma pulse. The estimate of the kinetic energy is systematically larger than the measured diamagnetic energy, which is also seen in a set of experimental programs from March 2016 (figure 7(b)). For these measurements a mean relative deviation of about $10 \%$ is found. In this context both the kinetic and diamagnetic energy values have been corrected for several uncertainties: $W_{d i a}^{\text {corr }}=1.08 W_{d i a}$ and $W_{k i n}^{\text {corr }}=0.8 W_{k i n}$, as explained in the following. The reason for the correction of the diamagnetic energy originate from the comparison of predicted and measured diamagnetic flux as discussed in section 2.2. Error sources for the correction of the kinetic energy are the density measurement, the evolution of plasma impurities and the mapping of the measured profiles from real space to flux surface coordinates. It is noted that in the context of these investigations predominantly isotropic plasma pressure can be assumed. Effects on the energy comparison due to a sensitivity of the Thomson scattering diagnostic measurement to perpendicular or parallel pressure components are negligible (detailed analysis in [30]).

\section{Summary and future plans}

The diamagnetic energy has been measured routinely with high accuracy during the second half of the first operational phase of W7-X. A compensation of external magnetic flux contributions has been successfully implemented using a set of calibrated compensation coils located in the vicinity of the diamagnetic loop.

A preliminary analysis of eight Rogowski coil segment signals reveals, as theoretically expected, that the diamagnetic energy and the dipole moment of the associated reconstructed Pfirsch-Schlüter current density distribution are proportional to each other.

MHD-equilibrium reconstruction based on measured pressure profiles and magnetic 
fluxes within the MINERVA framework [25] is under preparation. Via direct comparisons with free-boundary calculation results from VMEC [32] a more detailed description of the current distribution in the plasma is thought to be possible.

For future operational phases, it is planned to utilize the diamagnetic energy as an online safety interlock to shut off plasma heating systems (e.g. ECRH) within less than $50 \mathrm{~ms}$ to protect the vacuum vessel and critical components inside the vacuum vessel from damage. An algorithm is currently being tested, which compares the raw signals of the triangular-shaped diamagnetic loop and the surrounding four compensation coils with a given threshold value to identify a plasma formation failure or a sudden, unwanted plasma collapse and issues a status signal every $1 \mathrm{~ms}$. The algorithm will be implemented directly on the Field Programmable Gate Array (FPGA) unit of the measurement board. The ultimate goal is to use the status signal within the central safety system of W7-X to generate an interlock for the heating systems.

\section{Acknowledgements}

This work has been carried out within the framework of the EUROfusion Consortium and has received funding from the Euratom research and training programme 20142018 under grant agreement No 633053. The views and opinions expressed herein do not necessarily reflect those of the European Commission.

\section{References}

[1] C. Beidler et al , Fusion Technol., 17, 148 (1990)

[2] H.-S. Bosch et al , Nucl. Fusion 53, 126001 (2013)

[3] V.D. Shafranov, Plasma Equil. in a Magn. Field, Rev. of Plasma Phys. 2, 103 (1966)

[4] G. Grieger et al, Phys. Fluids B 4, 2081 (1992)

[5] J. Nührenberg and R. Zille, Phys. Lett. A 114, 129 (1986)

[6] T. Sunn Pedersen et al , Nat. Commun. 7, 13493 (2016)

[7] T. Sunn Pedersen et al , Nucl. Fusion 55, 126001 (2015)

[8] T. Sunn Pedersen et al, Phys. Plasmas 24, 055503 (2017)

[9] T. Klinger et al , Plasma Phys. Control. Fusion 59, 014018 (2017)

[10] R. Wolf et al , Nucl. Fusion 57, 102020 (2017)

[11] M. Hirsch et al , Nucl. Fusion 57, 086010 (2017)

[12] K. Rahbarnia et al , 43 ${ }^{\text {rd }}$ EPS Conference on Pl. Phys., Leuven, Belgium, P4.011 (2016)

[13] M. Endler et al , Fusion Eng. and Design, 100, 468 (2015)

[14] S. Besshou et al , Nucl. Fusion, 26, 1339 (1986)

[15] S.P. Hirshman and J.C. Whitson, Phys. Fluids 12, 3553 (1983)

[16] S.P. Hirshman et al , Comp. Phys. Comm., Vol. 431, 143 (1986)

[17] H.J. Gardner, Nucl. Fusion 8, 1417 (1990)

[18] B.B. Carvalho et al , $11^{\text {th }}$ IAEA Technical Meeting on Control, Data Acquisition, and Remote Participation for Fusion Research, Greifswald, Germany(2017), P2/3/03

[19] A. Werner, Rev. Sci. Instrum., 77, 10E307 (2006)

[20] V. Erckmann et al , Fusion Sci. Technol., 52, 291 (2007)

[21] G. Weir et al , 43 ${ }^{\text {rd }}$ EPS Conference on Pl. Phys., Leuven, Belgium, P4.009 (2016)

[22] J. Knauer et al , $43^{\text {rd }}$ EPS Conference on Pl. Phys., Leuven, Belgium, P4.017 (2016)

[23] W.H. Press et al , Num. Recipes, 3rd Edition, chp. 15, Cambridge Univ. Press (2007) 
[24] J.C. Schmitt et al , Phys. Plasmas, 21, 092518 (2014)

[25] J. Svensson and A. Werner, IEEE Int. Symp. on Intelligent Sign. Process., 1 (2007)

[26] J. Schilling et al , Working title: Magnetic equilibrium reconstructions at W7-X within the MINERVA framework, to be published in Nuclear Fusion (2018)

[27] E. Pasch et al , Rev. Sci. Instrum., 87, 11E729 (2016)

[28] S.A. Bozhenkov et al, Thomson scattering diagnostics at Wendelstein $7-X$ and its performance in the first operation phase, to be published in Journal of instrumentation (2018)

[29] N. Pablant et al , $43^{\text {rd }}$ EPS Conference on Pl. Phys., Leuven, Belgium, P4.013 (2016)

[30] G. Fuchert et al , Global energy confinement in the initial limiter configuration of Wendelstein $7-X$, to be published in Nuclear Fusion (2018)

[31] H. Yamada et al , Nucl. Fusion, 45, 1684 (2005)

[32] S.P. Hirshman et al , Comput. Phys. Commun. 43, 143 (1986) 


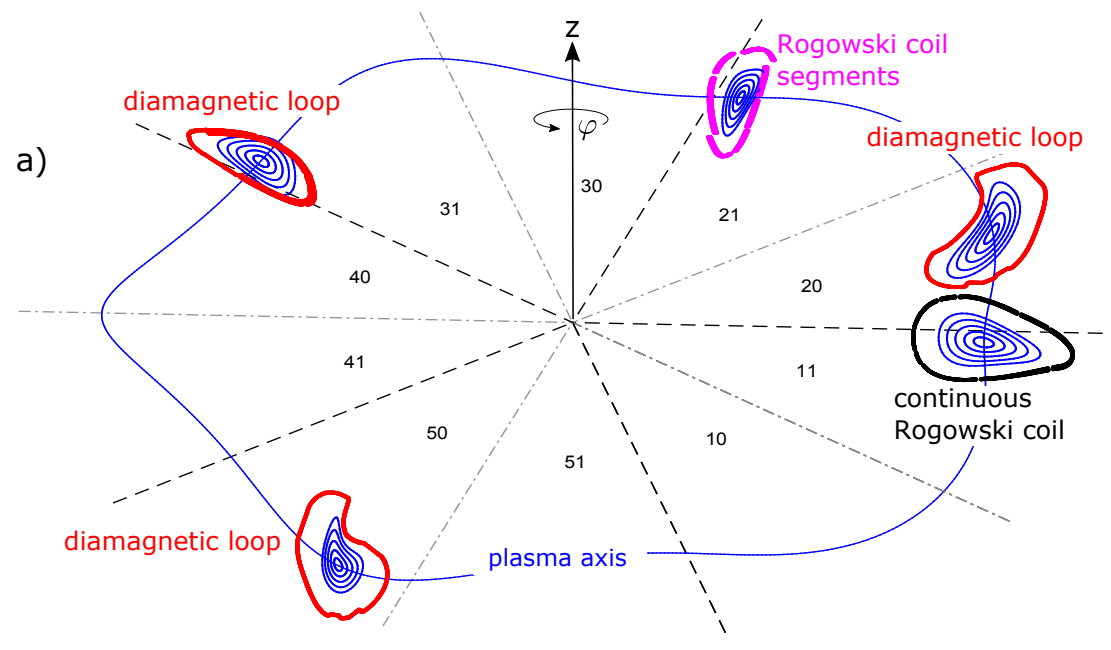

b)
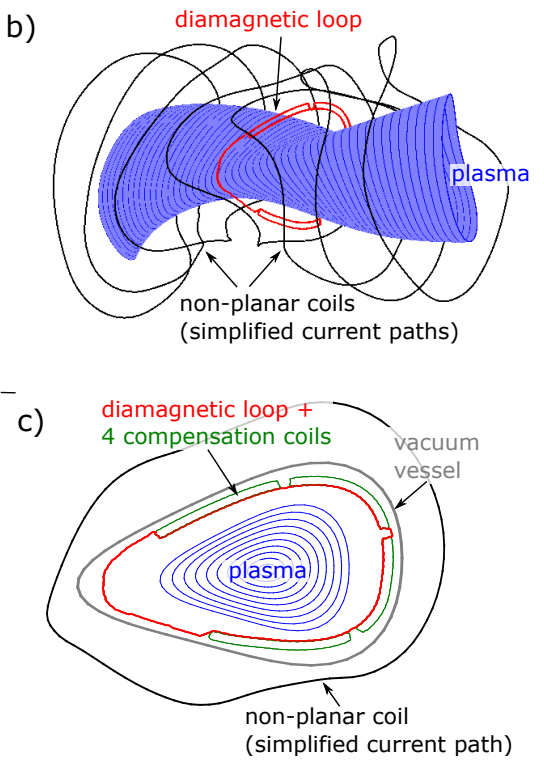

Figure 1. (color online) (a) Overview of location of magnetic diagnostics (diamagnetic loops(red), continuous Rogowski coil (black), Rogowski coil segments (magenta)) with flux surfaces (blue) at the corresponding toroidal locations and with the path of the magnetic axis (blue). The numbering of the half modules (HM) are included. (b) 3D-view of the diamagnetic loop (red) with compensation coils enclosing plasma (blue) and with vacuum magnetic field coils as filaments (black). (c) 2-D-view of diamagnetic loop (red) with compensation coils (green) in the plane of the loop with vacuum vessel (grey) and projected, simplified non-planar coil (black).

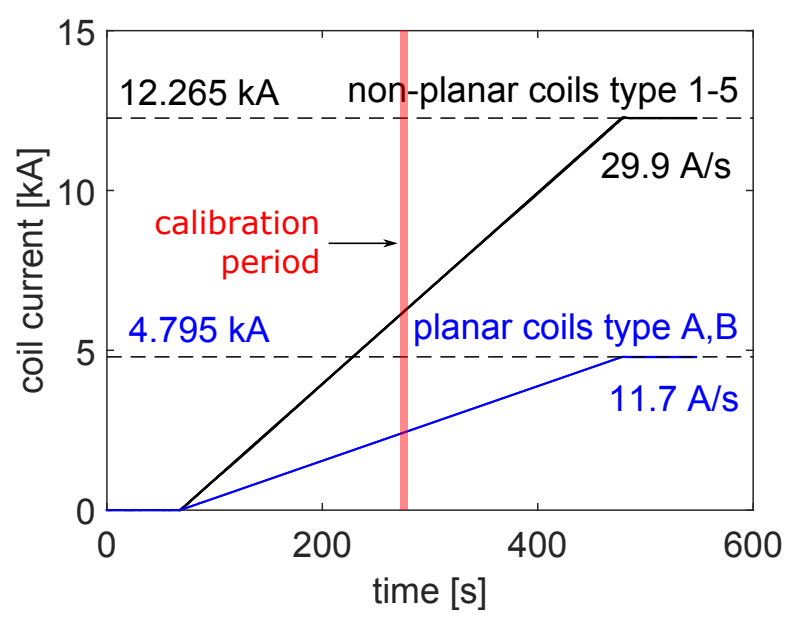

Figure 2. (color online) Simultaneously driven current-ramps within in the non-planar coils (black line) and planar coils (blue line). A time interval of $2 \mathrm{~s}$, indicated by the red bar, is used for the calibration of the compensation coils. 
a)

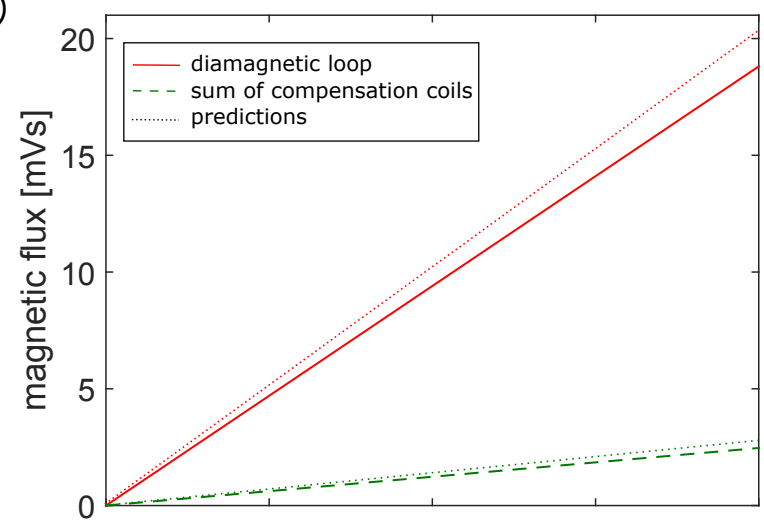

b)

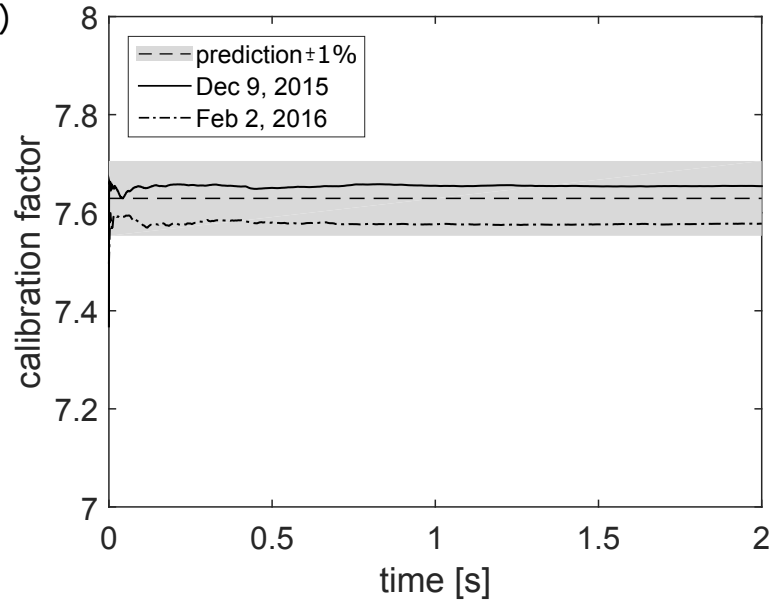

Figure 3. (color online) (a) Integrated magnetic fluxes within the calibration time interval of $2 \mathrm{~s}$ indicated in figure 2: diamagnetic loop in red (solid: measurement, dotted: prediction); sum of compensation coils in green (dashed: measurement, dotted: prediction. This measurement was taken on December $9^{t h}, 2015$. (b) Ratio of diamagnetic signal to sum of compensation signals: dashed: prediction (with $\pm 1 \%$ as gray shaded area), solid and dash-dotted: measurements from December $9^{t h}, 2015$ and February $2^{\text {nd }}, 2016$, respectively. 


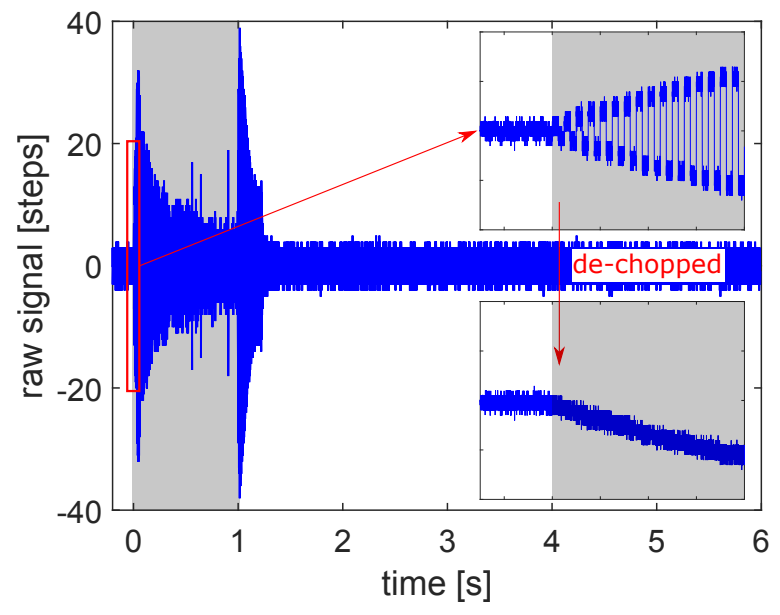

Figure 4. (color online) Typical raw signal of the triangular-shaped diamagnetic loop (W7-X program identifier, PID 20160308.012). The gray-shaded area indicates the time with plasma heating. The upper right zoomed insert shows the chopped raw signal, which is de-chopped (lower right insert) for further data evaluation. 

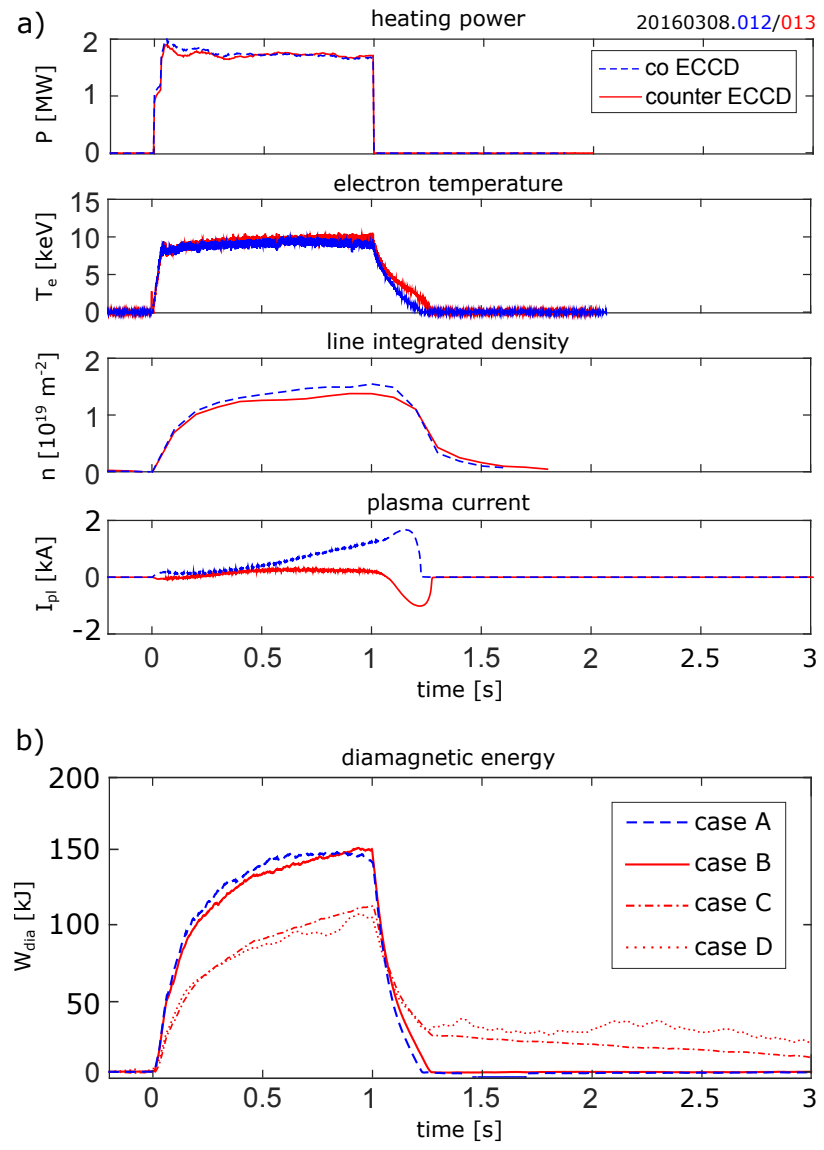

Figure 5. (color online) (a) Plasma parameter for co- (PID 20160308.012, blue dashed lines) and counter-ECCD (PID 20160308.013, red solid lines) are shown. (b) case A (blue dashed line, co-ECCD): the compensated energy signal measured by the triangular-shaped loop (HM 31, figure 1(a)), case B (red solid line, counterECCD): the compensated energy signal measured by the triangular-shaped loop, case $\mathrm{C}$ (red, dash-dotted line, counter-ECCD): the uncompensated energy signal measured by the triangular-shaped loop and case D (red, dotted line, counter-ECCD): the uncompensated energy signal measured by the bean-shaped loop (HM 20, figure 1(a)) 


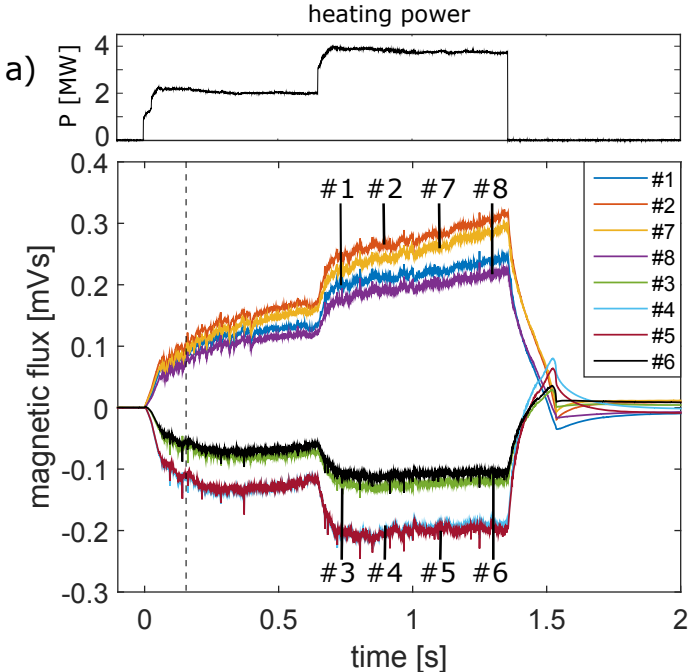

b)

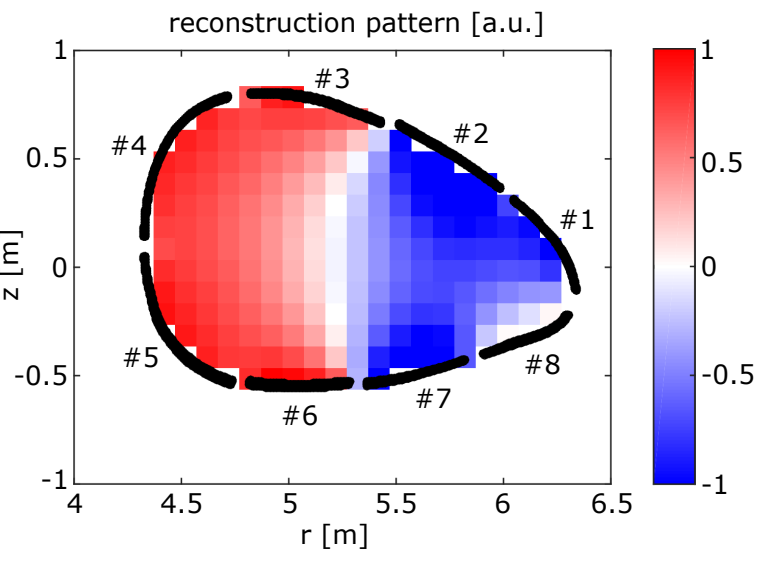

c)

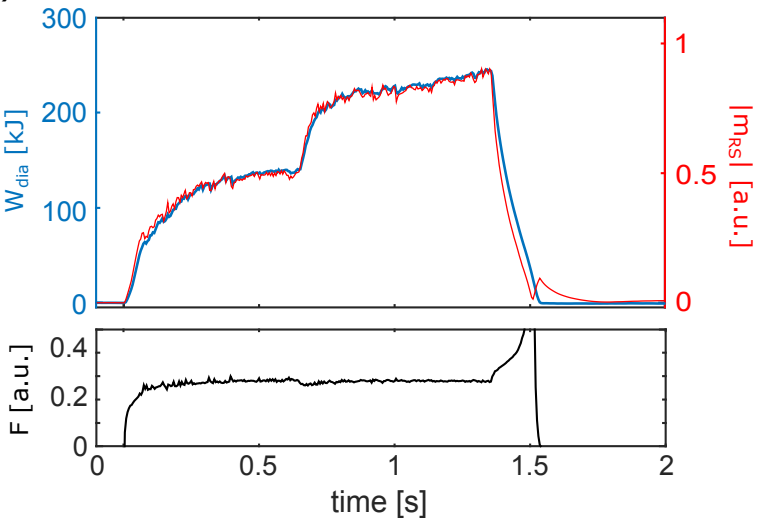

Figure 6. (color online) Rogowski coil segment signals for the plasma pulse PID 20160303.005 have been analysed. (a) top plot: ECR heating power, bottom plot: measured signals of eight individual segments (HM 21, figure 1(a)), The vertical dotted line indicates the time step $t=150 \mathrm{~ms}$, at which the signal pattern in figure $6(\mathrm{~b})$ has been reconstructed. (b) reconstructed signal pattern at $t=150 \mathrm{~ms}$ in the triangularshaped cross section, individual segments are indicated by numbers $\# 1 \ldots \# 8$, (c) top plot: The diamagnetic energy (blue line) is proportional to the associated dipole moment (red line) as described in the text. bottom plot: The proportionality factor during the plasma pulse is almost constant. 

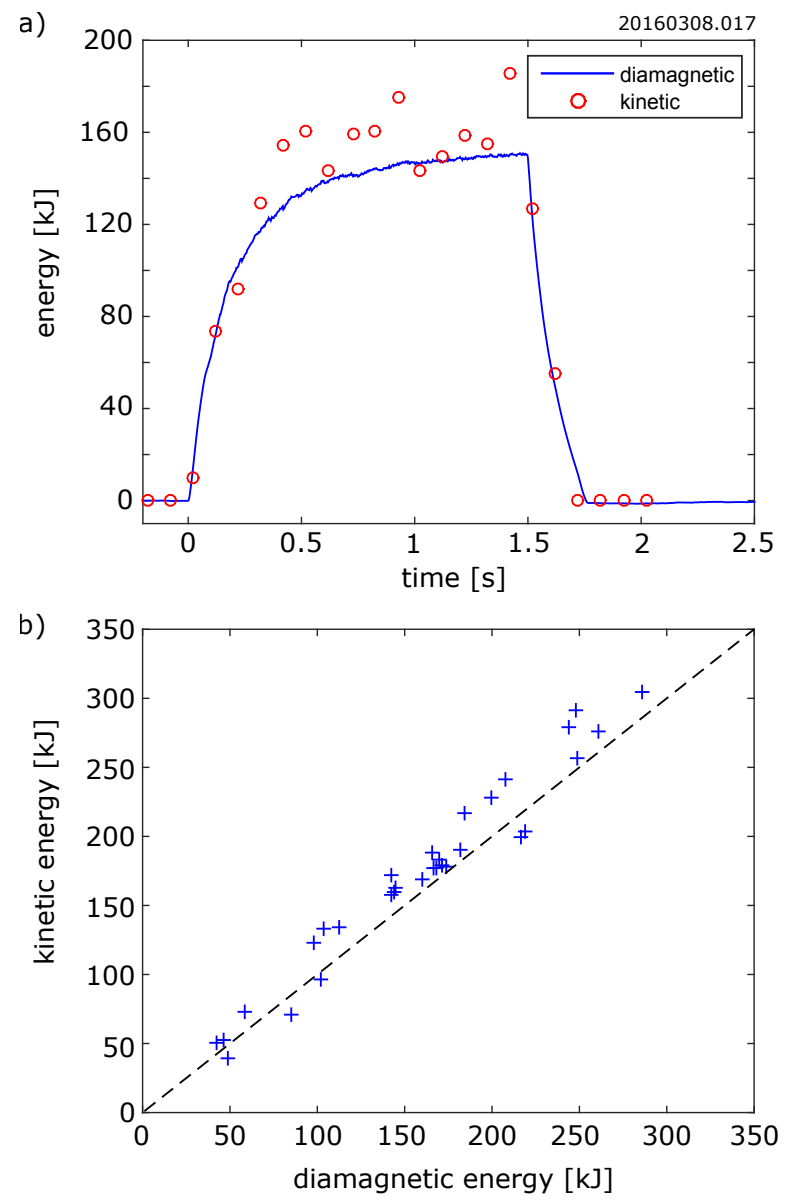

Figure 7. (color online) A direct comparison of the corrected kinetic (red circles) and diamagnetic energy (blue solid line) is shown for an individual plasma pulse PID 20160308.017 (a) and for a set of experimental programs from March 2016 (b). 